\section{AB0927 RELIABILITY AND VALIDITY OF TURKISH VERSION OF FIBROMYALGIA PARTICIPATION QUESTIONNAIRE}

L. Altan ${ }^{1}$, R. Çeliker ${ }^{2}$, I. Ercan ${ }^{3}$, M. Birtane ${ }^{4}$, K. Akgün ${ }^{5}$, C. Zateri ${ }^{6}$, N. Taştekin ${ }^{7}$, A. Rezvani ${ }^{8}$, I. Aktaş ${ }^{9}$, S. Özdolap ${ }^{10}$, E. Dursun ${ }^{11}$, N. Dursun ${ }^{11}$, S. Sarıkaya ${ }^{10}$. ${ }^{1}$ Physical Medicine and rehabilitation, Uludağ University Medical Faculty, Bursa; ${ }^{2}$ Physical Medicine and rehabilitation, Acibadem University, Faculty of Medicine, Istanbil; ${ }^{3}$ Uludağ University Medical Faculty, Bursa; ${ }^{4}$ Trakya University, Faculty of Medicine, Edirne; ${ }^{5}$ Istanbul University, Cerrahpaşa Faculty of Medicine, Istanbul; ${ }^{6}$ Physical Medicine and rehabilitation, Canakkale Onsekiz Mart University, Faculty of Medicine, Çanakkale, ${ }^{7}$ Physical Medicine and rehabilitation, Trakya University, Faculty of Medicine, Edirne; ${ }^{8}$ Physical Medicine and rehabilitation, Bezmialem University, Faculty of Medicine; ${ }^{9}$ Physical Medicine and rehabilitation, Fatih Sultan Mehmet Teaching and Research Hospital, Istanbul; ${ }^{10}$ Physical Medicine and rehabilitation, Bulent Ecevit University, Faculty of Medicine, Zonguldak; ${ }^{11}$ Physical Medicine and rehabilitation, Kocaeli University, Faculty of Medicine, Kocaeli, Turkey

Background: Fibromyalgia (FMS) is a chronic health problem characterized by a wide range of physical and psychological symptoms. There are few high-quality instruments to evaluate the participation and social functioning of fibromyalgia patients. Farin et al. designed the Fibromyalgia Participation Questionnaire (FPQ) as an instrument for measuring the participation and social functioning of FMS patients. The original version of FPQ has been demonstrated to have acceptable internal consistency, reliability and criterion validity.

Objectives: To test reliability and validity of Turkish version of Fibromyalgia Participation Questionnaire (FPQ-T)

Methods: One hundred and eighty-four female fibromyalgia syndrome patients were included in the study. All patients filled FPQ-Turkish (FPQ-T) questionnaire which was obtained by translation from German according to the guideline for the cross-cultural adaptation process.

The patients filled the revised Fibromyalgia Impact Questionnaire (FIQ) and reevaluated FPQ-T two hours later. Internal consistency reliability of FPQ-T was assessed by calculating "if item deleted" using Cronbach alpha and "item-total correction" coefficient for each item of the questionnaire. Consistency of subscales and correlation of test-retest values were assessed. Test-retest values were compared using Wilcoxon test. Criterion validity was measured using FIQ scales by Spearman's rho correlation coefficient.

Results: For internal reliability, Cronbach alpha coefficient was calculated as 0.957 for non-working and 0.958 for working patients. Cronbach alpha values of $0.939,0.871$, and 0.914 were obtained for daily, social, and work life, respectively. Correlation coefficients were 0.888 for daily life, 0.859 for social life, and overall 0.901 in non-working group versus 0.896 in working group. Comparison of scores obtained from test-retest measurements showed no significant difference except for Item-3. Correlation of symptom severity score (SSS) and FPQ-T were $r=0.385$ $(p<0.001)$ and $r=0.390(p<0.001)$ for the non-working and working sub-groups, respectively. Construct validity evaluation showed significant correlation between SSS and FPQ-T.

Conclusions: The results of our study showed that FPQ-T is reliable and valid for assessing participation and social functioning in fibromyalgia patients in our society.

References:

[1] Williams DA, Clauw DJ (2009) Understanding fibromyalgia: Lessons from the broader pain research community. J Pain 10:777-791.

[2] Farin E, Ullrich A, Hauer J (2013) Participation and social functioning in patients with fibromyalgia: development and testing of a new questionnaire. Health Qual Life Outcomes 11:135.

Disclosure of Interest: None declared

DOI: 10.1136/annrheumdis-2017-eular.1710

\section{AB0928 FATIGUE IN FIBROMYALGIA REFLECTS OVERALL BURDEN OF DISEASE}

M. Antivalle, A. Batticciotto, F. Rigamonti, M.C. Ditto, M.C. Gerardi, R. Talotta, A. Mutti, P. Sarzi-Puttini. Rheumatology, L. Sacco University Hospital, Milano, Italy

Background: Fatigue in fibromyalgia is a very common and disabling symptom, being reported by over $75 \%$ of patients. However, the relationship between fatigue and the different manifestations of fibromyalgia has not been convincingly elucidated.

Objectives: Aim of the our work was to study the relationship between fatigue, pain, and somatic symptoms in fibromyalgia.

Methods: 123 patients with fibromyalgia classified according to 2010 criteria were studied, 113 Females and 10 Males, mean age $47.41 \pm 11.21$ years, mean disease duration $6.83 \pm 6.52$ years. Fatigue was assessed by FACIT-Fatigue questionnaire (13 items, score $0-52$, higher values representing less fatigue), and results were correlated to the number of Tender points (TP), to Widespread Pain Index (WPI), to Symptom severity Scale (SSS), and to Polysymptomatic Distress Scale (PDS) values. Correlations were computed by parametric or non parametric methods as appropriate.

Results: Average FACIT-Fatigue score $(22.51 \pm 10.6)$ was lower than the 25th percentile of the general population (35), and was not correlated to age or to disease duration $(p>0.05)$. FACIT-Fatigue showed statistically significant inverse
Fig. 1. Left panel: FACIT-Fatigue score according to the presence or absence of individual somatic symptoms.

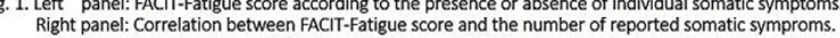
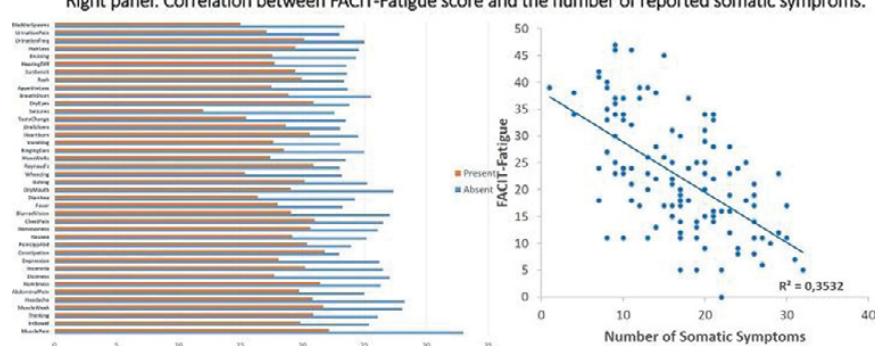

correlations with the number of tender points $(r=-.303, p=0.001)$, WPI score $(r=-0.329, p<0.001)$, SSS score $(r=-0.651, p<0.001)$, PSD score $(r=-0.522$, $p<0.001)$, and with the total number of somatic symptoms $(r=-0.594, p<0.000)$ (Fig. 1, right panel). Furthermore, for each of the 40 somatic symptoms suggested by 2010 criteria, the presence of the symptoms was associated with higher levels of fatigue (Fig 1, left panel).

Conclusions: The results of our study confirm that fatigue is a prominent feature of fibromyalgia. Higher levels of fatigue reflect higher levels of widespread pain, and a higher burden of somatic symptoms.

Disclosure of Interest: None declared

DOI: 10.1136/annrheumdis-2017-eular.6534

\section{AB0929 BALANCE IN FIBROMYALGIA IS NOT RELATED WITH LOWER LIMB STRENGTH}

P. Serra-Añó, M. Aguilar-Rodríguez, S. Cortés-Amador, G.V. Espí-lópez, N. Sempere-Rubio. Physical Therapy, University of Valencia, Valencia, Spain

Background: Postural control, that depends on many sensory contributors and motor skills, is altered in people with fibromyalgia (FM) ${ }^{1-3}$. Knowing those contributors that could be impaired in this population is important to design an appropriate balance therapy.

Objectives: Due to the fact that people with FM use to present a strength loss ${ }^{7}$, our main goal was to explore the relation between postural control and lower limb strength in this population. Further, the sensory inputs contributors to the postural control were assessed.

Methods: A cross-sectional study was conducted in a sample of $40 \mathrm{FM}$ patients. A force platform was used to assess postural control under five bipedal conditions: i) eyes-open (EO); ii) dual task (recalling a typical day in their life) (DT); iii) eyesclose (EC); iv) eyes-open over a foam (FEO); v. eyes-close over a foam (FEC). Three variables, "Ellipse", "RMS" and "Sample Entropy" (SampEn), were recorded (for both directions). Further, maximal quadriceps (qdc) and hamstring (hmsg) isometric strength were assessed using a dynamometer. Spearman correlations were conducted to establish the relationship between postural control and strength and a repeated-measures MANOVA, to analyze differences between conditions. Results: When EO and DT (performed with eyes open) were compared, no significant differences were obtained. However when patients closed their eyes, an increase in ellipse and RMS_AP and a decrease in variability were obtained. The same was true when the tests were performed over a foam (altering proprioception), Table 1.

Table 1. Comparison of sensory inputs results

\begin{tabular}{lccccc}
\hline & Ellipse $\left(\mathrm{mm}^{2}\right)$ & RMS_AP $(\mathrm{mm})$ & RMS_ML $(\mathrm{mm})$ & SampEn_AP & SampEn_ML \\
\hline EO & $295.66(460.93)$ & $5.58(3.66)$ & $2.46(2.30)$ & $0.74(0.14)$ & $0.90(0.10)$ \\
DT & $388.92(530.23)$ & $5.77(4.22)$ & $2.79(2.18)$ & $0.67(0.15)$ & $0.86(0.13)$ \\
EC & $508.18(801.69)^{1}$ & $6.77(3.94)^{12}$ & $3.08(2.04)$ & $0.65(0.15)^{1}$ & $0.85(0.14)^{1}$ \\
FEO & $815.32(981.44)^{123}$ & $7.63(4.19)^{12}$ & $4.81(3.04)^{123}$ & $0.61(0.14)^{12}$ & $0.79(0.10)^{123}$ \\
FEC & $1425.54(1291.53)^{1234}$ & $10.87(4.20)^{1234}$ & $6.48(2.95)^{1234}$ & $0.53(0.11)^{1234}$ & $0.69(0.13)^{1234}$ \\
\hline
\end{tabular}

EO: eyes-open; DT: dual task; EC: eyes-close; FEO: Eyes-open over a foam; FEC: Eyes-close over a foam; Superscript 1,2, 3, 4: Significant differences with test $1,2,3$ and 4 , respectively $(p<0.05)$.

Furthermore, no significant correlations $(p>0.05)$ were observed between strength and balance except for a weak significant correlation between hmsg strength and RMS_AP $\left(r_{s}=-0.33, p<0.05\right)$ in the EC test.

Conclusions: Postural control worsens in FM people when some sensory inputs are altered or missed. Further, there is no correlation between lower limb strength and balance in this population.

\section{References:}

[1] Akkaya N, Akkaya S, Atalay NS, Acar M, Catalbas N, Sahin F. Assessment of the relationship between postural stability and sleep quality in patients with fibromyalgia. Clin Rheumatol. 2013;32(3):325-331.

[2] Jones KD, King LA, Mist SD, Bennett RM, Horak FB. Postural control deficits in people with fibromyalgia: a pilot study. Arthritis Res Ther. 2011;13(4):R127.

[3] Russek LN, Fulk GD. Pilot study assessing balance in women with fibromyalgia syndrome. Physiother Theory Pract. 2009;25(8):555-565.

[4] Góes SM, Stefanello JM, Homann D, Lodovico A, Hubley-Kozey CL, Rodacki $\mathrm{AL}$. Torque and muscle activation impairment along with insulin resistance are associated with falls in women with fibromyalgia. J Strength Cond Res Strength Cond Assoc. 2016; 30(11), 3155-3164. 
Acknowledgements: Grant GV2016/140. Conselleria Educació Ciencia i esports. Valencia, Spain.

Disclosure of Interest: None declared

DOI: 10.1136/annrheumdis-2017-eular.3433

\section{AB0930 CROSS-SECTIONAL ANALYSIS OF THE AUTONOMIC NERVOUS SYSTEM (HEART RATE VARIABILITY): CORRELATIONS WITH PSYCHOLOGICAL DIMENSIONS IN WOMEN WITH FIBROMIALGIA, RHEUMATOID ARTHRITIS AND HEALTHY CONTROLS}

T.L. Santiago, D. Peixoto, C. Costa, J.A. Pereira da Silva. Rheumatology, Centro Hospitalar e Universitário de Coimbra, Coimbra, Portugal

Background: Autonomic nervous system (ANS) dysfunction has been proposed to play a role in the pathophysiology and maintenance of rheumatic diseases, including fibromyalgia (FM) and rheumatoid arthritis (RA). Heart rate variability (HRV) analyses provide a quantitative marker of ANS activity. Some studies suggest an association between reduced HRV parameters and psychological dimensions, namely a negative emotional state. This led us to hypothesize an association between rheumatic diseases and higher sympathetic activity mediated by a negative emotional state.

Objectives: To establish correlates between HRV parameters with rheumatic disease groups and psychological dimensions.

Methods: Sixty women (FM, $n=20$; RA, $n=20$; healthy controls (Ct), $n=20$ ) completed a self-reported questionnaire addressing demographic characteristics, the Eysenck Personality Questionnaire, the Hospital Anxiety and Depression Scale, and the Beck Depression Inventory-II (BDI-II).

HRV analysis was performed by photopletismography between 8:00 and 10:00am, after an overnight fast, in a sitting position, for 5-minutes. We obtained the time and frequency-domain indices of HRV, including SDNN (standard deviation of the NN intervals), RMSSD (root-mean square differences of successive R-R intervals), high frequency power (HF), low frequency power (LF) and very low frequency (VLF). LF/HF ratio reflects sympathetic to parasympathetic balance. Statistical analysis were performed considering: A) Rheumatic disease groups (FM/RA/Ct), and B) Psychological scores (irrespective of disease group): higher versus lower tertile in the personality questionnaires and score above (depression) versus below 20, in BDI-II. Between-groups comparisons were performed with Kruskal-Wallis test and analysis of covariance (age was adjusted during analyses), as appropriate.

Results: Neuroticism, anxiety and depression scores were significantly higher in FM and RA patients compared with controls $(p<0.05)$. However, no statistically significant difference was observed in HRV parameters between disease groups. No statistically significant difference was observed in HRV parameters between tertile groups for psychological dimensions, except for depression. The values of $\mathrm{HF}$ power (parasympathetic activity) were lower in the high depression group compared to the low depression group $(p<0.05)$. The ratio of $L F / H F$ was higher among the depression group than the control group $(p<0.05)$.

Conclusions: This study did not found significant differences in the HRV between the three rheumatic disease groups. The results confirm that depression is accompanied by dysfunction of the autonomic nervous system, specifically lower parasympathetic activity. These results suggest that psychological dimensions, namely depression, must be taken into account when evaluating the ANS and its impact in disease pathogenesis.

Disclosure of Interest: None declared

DOI: 10.1136/annrheumdis-2017-eular.5974

\section{AB0931 EFFECTS OF MANUAL THERAPY ON PAIN, POSTURE, FLEXIBILITY, QUALITY OF SLEEP AND DEPRESSIVE SYMPTOMS IN FIBROMYALGIA SYNDROME}

T. Duymaz ${ }^{1}$, G. Sakınç Toprak ${ }^{2}{ }^{1}$ Physiotherapy and Rehabilitation, Istanbul Bilim University, Şişli-Istanbil; ${ }^{2}$ Physiotherapy and Rehabilitation, Acıbadem Hospital, Izmit-Kocaeli, Turkey
Background: Fibromyalgia syndrome (FMS) is a chronic musculoskeletal pain condition characterized by widespread and a constellation of other symptoms. Objectives: The purpose of this study was to investigate the therapeutic effects of a Manual Therapy (MT) protocol for improving pain, posture, flexibility, tender points, impact of FMS symptoms, sleep quality and depressive symptoms with FMS.

Methods: Patients completed demographic information, a number of self-report measures including Visual Analog Scale for assessing pain, Fibromyalgia Impact Questionnaire, the Pittsburgh Quality of Sleep Questionnaire Index and the Hospital of Anxiety and Depression Scale.The patients underwent a protocol of MT for a 60 -minute session for 3 weeks (5/wk). The protocol was as the following release and mobilisation. All analyses were performed with the SPSS (version 22.0)statistical program

Results: 16 women,3 men patients who had FMS were treated with MT.Demographic variables of the participants are provided in Table 1. While there was a favorable change in all measurement parameters after treatment $(p<0.001)$. There was a statistically significant difference between before and after the treatment measurement parameters (Table 2)

Table 1.Demographic variables of the participants

\begin{tabular}{lc}
\hline Variables & Mean \pm SD $(\min -\max )$ \\
\hline Age $($ year) & $36.05 \pm 8.16(20-58)$ \\
BMl $\left(\mathrm{kg} / \mathrm{m}^{2}\right)$ & $24.19 \pm 4.16(19.49-33.06)$ \\
Duration of symptoms (year) & $4.81 \pm 2.41(1-9)$ \\
\hline
\end{tabular}

Conclusions: This study found that the application of a MT protocol was effective for improving pain, posture, flexibility,tender points, impact of FMS symptoms, sleep quality and depressive symptoms in individuals with FMS.

References:

[1] Goldenberg DL, Burckhardt C, Crofford L. Management of fibromyalgia syndrome. JAMA. 2004;292:2388-2395.

[2] Castro-Sa' nchez AM, Matara' n-Peñ arrocha GA, Arroyo-Morales M. Effects of myofascial release techniques on pain, physical function, and postural stability in patients with fibromyalgia: a randomized controlled trial. Clin Rehabil. $2011 ; 25: 800-813$

Disclosure of Interest: None declared

DOI: 10.1136/annrheumdis-2017-eular.5833

\section{AB0932 PREVALENCE OF TYPE D PERSONALITY IN TURKISH PATIENTS WITH FIBROMYALGIA SYNDROME}

Y. Garip ${ }^{1}$, T. Guler ${ }^{1}$, O.B. Tuncer ${ }^{2}$, S. Onen ${ }^{3} .{ }^{1}$ Physical Medicine and Rehabilitation, Ankara Numune Training and Research Hospital, Ankara; ${ }^{2}$ Physical Medicine and Rehabilitation, Medipol University Medical School, Istanbul; ${ }^{3}$ Psychiatry, Sevket Yılmaz Eğitim ve Araştırma Hastanesi, Bursa, Turkey

Background: Type D personality is a distressed personality type involving two personality traits, namely negative affectivity and social inhibition, which are described as tendency to experience negative emotions and to inhibit self-expression in social relationships, respectively (1).

Objectives: The present study investigated the prevalence of type $D$ personality in Turkish patients with fibromyalgia (FM) and evaluated the association between type D personality and clinical parameters of FM. Although there is adequate number of studies focusing on the relation between FM and psychological conditions such as depression and anxiety; this topic has been rarely addressed in the literature.

Methods: A total of 100 patients with FM fulfilling 1990 American College of Rheumatology (ACR) diagnostic criteria and 50 healthy controls were included. Type D personality was assessed by Type D Scale-14 (DS-14). FM disease severity was determined by Fibromyalgia Impact Questionnaire (FIQ), functional status by Stanford Health Assessment Questionnaire (HAQ), and health-related quality of life (HRQoL) by Nottingham Health Profile (NHP). Severity of pain and fatigue were measured by Visual Analog Scale (VAS).

Abstract AB0931 - Table 2. Comparison of before and after treatment in all measurement parameters

\begin{tabular}{|c|c|c|c|c|}
\hline Variables & $\begin{array}{c}\text { Before Treatment } \\
\text { Mean } \pm \text { SD (min-max) }\end{array}$ & $\begin{array}{c}\text { After Treatment } \\
\text { Mean } \pm \text { SD (min-max) }\end{array}$ & $\mathrm{z}$ & $\mathrm{p}$ \\
\hline Number of tender points & $12.10 \pm 1.24(10-15)$ & $7.31 \pm 2.26(2-11)$ & -3.840 & $0.000^{* *}$ \\
\hline VAS at night & $6.84 \pm 1.30(3-9)$ & $0.91 \pm 0.64(0-2)$ & -3.843 & $0.000^{\star \star}$ \\
\hline VAS in activity & $7.31 \pm 1.24(4-9)$ & $1.11 \pm 0.81(0-2.20)$ & -3.850 & $0.000^{\star *}$ \\
\hline VAS in rest & $6.42 \pm 1.46(3-9)$ & $0.49 \pm 0.81(0-2.40)$ & -3.834 & $0.000^{\star *}$ \\
\hline Distance of Tragus-Wall $(\mathrm{cm})$ & $12.08 \pm 1.95(9-17)$ & $10.72 \pm 1.90(8.20-15.60)$ & -3.529 & $0.000^{* \star}$ \\
\hline Distance of processus spinosus-Skapula $(\mathrm{cm})(\mathrm{R})$ & $10.65 \pm 3.03(6.50-17)$ & $10.26 \pm 2.76(7-15)$ & -2.007 & $0.045^{\star}$ \\
\hline Distance of processus spinosus-Skapula $(\mathrm{cm})(\mathrm{L})$ & $11.21 \pm 3.17(7-17)$ & $10.26 \pm 2.76(7-15)$ & -2.842 & $0.004^{\star \star}$ \\
\hline Trunk Lateral Flexion $\left({ }^{\circ}\right)(\mathrm{R})$ & $36.47 \pm 3.35(30-41)$ & $37.00 \pm 3.39(30-41)$ & -1.821 & 0.069 \\
\hline Trunk Lateral Flexion $\left({ }^{\circ}\right)(\mathrm{L})$ & $33.47 \pm 4.01(27-41)$ & $36.68 \pm 3.28(30-41)$ & -3.534 & $0.000^{\star \star}$ \\
\hline Distance of hand-floor $(\mathrm{cm})$ & $6.86 \pm 4.84(0-17)$ & $5.18 \pm 3.92(0-14)$ & -3.219 & $0.001^{\star \star}$ \\
\hline $\mathrm{FIQ}$ & $56.25 \pm 18.74(19.79-85.58)$ & $46.39 \pm 17.99(19.59-67.98)$ & -3.724 & $0.000^{\star \star}$ \\
\hline HAD-A & $10.89 \pm 3.91(5-19)$ & $4.73 \pm 3.49(0-10)$ & -3.849 & $0.000^{\star *}$ \\
\hline HAD-D & $9.78 \pm 3.29(2-15)$ & $5.05 \pm 2.06(0-9)$ & -3.632 & $0.000^{\star \star}$ \\
\hline PSQI & $10.52 \pm 3.40(5-15)$ & $3.63 \pm 2.47(0-8)$ & -3.830 & $0.000^{\star \star}$ \\
\hline
\end{tabular}

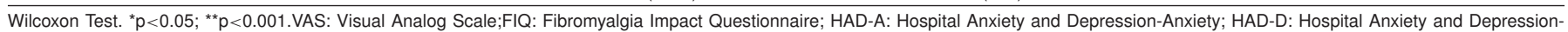
Depression; PSQI: Pittsburgh Quality of Sleep Questionnaire Index. 